\title{
Theoretical Study of Current-Voltage Characteristics of Electron-Hole Bilayer Tunnel Field Effect Transistors of Different Channel Semiconductors
}

\author{
P. WiŚnIEWSKI ${ }^{a, b, c, *}$ AND B. MAJKUSIAK ${ }^{b}$ \\ ${ }^{a}$ Centre for Advanced Materials and Technologies CEZAMAT, \\ Warsaw University of Technology, Poleczki 19, 02-822 Warsaw, Poland \\ ${ }^{b}$ Institute of Microelectronics and Optoelectronics, Warsaw University of Technology, \\ Koszykowa 75, 00-662 Warsaw, Poland \\ ${ }^{c}$ Center for Terahertz Research and Applications (CENTERA), \\ Institute of High-Pressure Physics, Polish Academy of Sciences, \\ Sokołowska 29/37, 01-142 Warsaw, Poland
}

Received: 21.08.2020 \& Accepted: 30.06.2021

Doi: $10.12693 /$ APhysPolA.140.186

*e-mail: piotr.wisniewski@pw.edu.pl

\begin{abstract}
In this paper, the current-voltage characteristics of electron-hole bilayer tunnel field effect transistors of different channel materials ( $\mathrm{Si}, \mathrm{Ge}, \mathrm{InAs}$ ) and various geometric parameters (channel thickness, oxide thickness) are modeled and discussed. We show that the structure studied in this work can exhibit very sharp turn-on transfer characteristics due to the quantum-mechanical tunneling as the transport mechanism. The theoretical model is based on the self-consistent solution of Poisson's equation and Schrödinger's equation with an effective mass approximation of the Hamiltonian. Direct and phonon-assisted interband tunneling currents are taken into account depending on the channel materials. We show that channel semiconductor parameters have a crucial impact on the properties of electron-hole bilayer tunnel field effect transistors which results from the fact that the energy band structure of the semiconductor determines the interband tunneling probability and current.
\end{abstract}

topics: 2D carrier gas, interband tunneling, steep-slope FET, field effect transistors

\section{Introduction}

The tunnel field effect transistors (TFETs) are a class of the steep-slope field effect transistors that are considered as possible successors of the metal-oxide-semiconductor field effect transistors (MOSFETs) [1, 2]. An electron-hole bilayer tunnel field effect transistor (EHB TFET) is a kind of a tunnel field effect transistor in which the interband tunneling happens between the electron and hole quantum wells induced at the opposite surfaces of a very thin semiconductor layer [3]. Figure 1 illustrates the geometrical structure and physics of such a transistor. The top (TG) and bottom (BG) gates overlap the drain region and the source region, which close the current paths between quantum wells and device terminals.

Owing to the non-thermionic nature of the tunnel transport mechanism [4-6], the gate voltage dependence of the current in the subthreshold range in TFETs is steeper than $60 \mathrm{mV} /$ decade - a theoretical limit for conventional MOSFETs at room temperature. The step-like nature of the densityof-states functions (DOS) in the electron and hole quantum wells and lack of heavy doping-induced band tails can additionally lower the subthreshold swing in EHB TFETs [7, 8].

A low subthreshold swing, high ON current, and high $\mathrm{ON} / \mathrm{OFF}$ current ratio appoint requirements on the transistor parameters [9-13] such as the gate insulator thickness, semiconductor thickness, etc. Also, a type of semiconductor material should have a crucial impact on the properties of the EHB TFET. The energy band structure of the semiconductor (the relative location of the conduction and valence band edges, the energy gap width, the electron and hole effective masses) determines the direct interband tunneling probability and current, and additionally, the effective masses determine the spectrum of discrete energy levels in the electron and hole quantum wells [14]. Some theoretical works analyze the heterostructure EHB TFET [15-17] and only a few experimental works investigate the bilayer TFET [18-21]. 


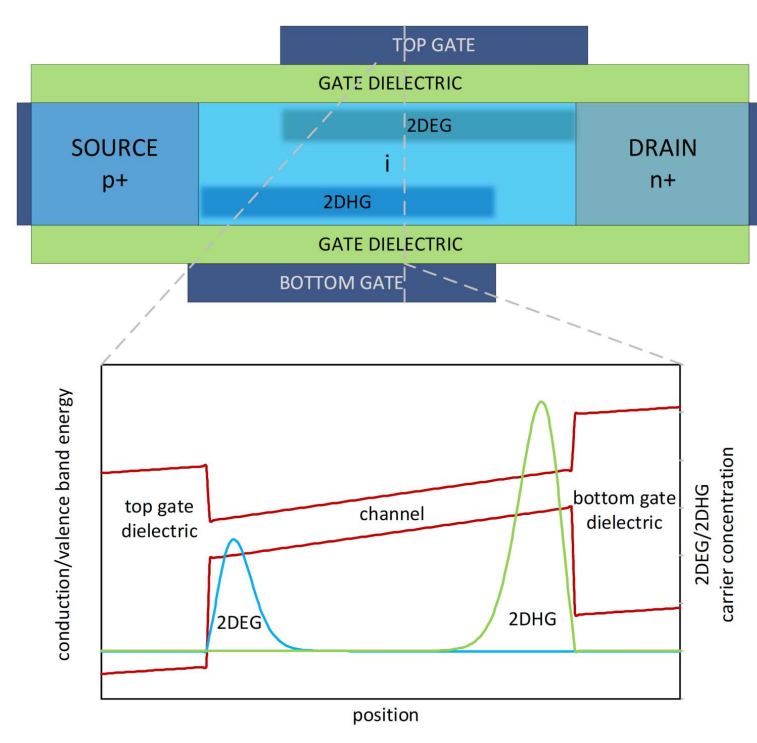

Fig. 1. Structure of the EHB TFET, the energy band diagram and the electron and hole concentration distributions in the vertical direction.

In this work, we model and analyze the currentvoltage characteristics of the EHB TFET for various channel materials ( $\mathrm{Si}, \mathrm{Ge}, \mathrm{InAs}$ ) and geometric parameters of the structure (channel thickness, oxide thickness). We calculate the position of energy levels in quantum wells, and based on this, we analyze the shape of the transfer characteristics of the Ge EHB TFET.

\section{Theory}

When appropriate potentials are applied to the gate electrodes, the two-dimensional electron gas (2DEG) and the two-dimensional hole gas (2DHG) are formed in the channel near the semiconductorgate oxide interfaces. Carrier transport occurs between the 2D gases, mainly in the gate overlap area in the confinement direction. The interband tunneling current is determined by the density-of-states function in the valence and conduction bands. It is assumed that holes and electrons in the channel are in equilibrium with the source and drain regions, respectively. Having the energy levels and the corresponding wave functions for different bands at given boundary conditions, the tunneling current is calculated.

The electrostatic model of the EHB TFET is based on the self-consistent solution of the 1D Poisson's equation and 1D Schrödinger's equation, and studied using the parabolic effective mass approximation (EMA) for holes and electrons. The system is described with

$$
\nabla(\varepsilon \nabla \varphi)=-q\left(p-n-N_{A}\right)
$$

and

$$
\left(\mp \frac{\hbar^{2}}{2} \nabla \frac{1}{m_{c / v}} \nabla+V_{c / v}\right) \Psi_{c / v}=E \Psi_{c / v}
$$

and solved in a cross-section of the simulated device, as illustrated in Fig. 1. Here, $\varphi$ is the potential, $\varepsilon$ is the electrical permittivity, $N_{A}$ is the acceptor concentration, and $n$ and $p$ are the electron and hole concentrations, respectively. The electron/hole effective masses are denoted by $m_{c / v}$, while $V_{c / v}$ are the conduction/valence band edge energies, $E$ is the total energy and $\Psi_{c / v}$ are the electron/hole wave functions.

The total band-to-band tunneling current is a sum of direct tunneling $J_{\text {dir }}$ and phonon-assisted tunneling $J_{\mathrm{ph}}$ currents. The direct interband tunneling current is modeled as the sum of contributions from different pairs of energy levels in the conduction and valence bands. Namely,

$$
J_{\text {dir }}=\frac{4 \pi q}{\hbar} \sum_{n} \sum_{k}|M|^{2} \operatorname{JDOS}_{2 \mathrm{D}}\left(f_{c}-f_{v}\right),
$$

where $f_{c}$ and $f_{v}$ are the Fermi-Dirac distribution functions for the conduction and valence bands, and $\mathrm{JDOS}_{2 \mathrm{D}}$ indicates the joint density-of-state function for the 2D-2D tunneling system. The following definition is applied:

$$
\mathrm{JDOS}_{2 \mathrm{D}}=\frac{m_{r}}{4 \pi \hbar^{3}} \Theta\left(E_{h h / l h, k}-E_{\Gamma, n}\right),
$$

where $m_{r}=2 m_{c} m_{v} /\left(m_{c}+m_{v}\right), E_{h h / l h, k}$ and $E_{\Gamma, n}$ are the energy levels of the positions of heavy/light holes and $\Gamma$-valley electrons, respectively.

Term $M$ in (3) is the coupling coefficient. It is calculated for the 2D-2D tunneling system using Bardeen's transfer Hamiltonian approach [22, 23] with the assumption of the conservation of the total energy and parallel momentum in the $2 \mathrm{D}-2 \mathrm{D}$ system. It can be written as

$$
|M|^{2}=\frac{\hbar^{2} E_{G}^{\Gamma}}{2 m_{r}} \delta_{k_{\perp}, k_{\perp}^{\prime}}\left|\Psi_{c, n} \Psi_{v, k}\right|^{2} C_{0}(\theta),
$$

where $k_{\perp}$ and $k_{\perp}^{\prime}$ are the transverse wave-vector components of electrons and holes $E_{G}^{\Gamma}$ is the direct bandgap of the material, and $C_{0}(\theta)$ is the form factor $[24,25]$ accounting for the dependence of the coupling element on the direction of the electric field.

The conservation of the total energy can be written as

$$
E_{h h / l h, k}-\frac{\hbar^{2} k_{\perp}^{\prime 2}}{2 m_{v}}=E_{\Gamma, n}+\frac{\hbar^{2} k_{\perp}^{2}}{2 m_{c}} .
$$

where $m_{v}$ is the effective mass of heavy/light hole, $m_{c}$ is the $\Gamma$-valley electron effective mass, and $k$ and $n$ indexes number the subband energy levels of holes and electrons, respectively. For $k_{\perp}=k_{\perp}^{\prime}$, (6) determines the tunneling energy for each pair of electron and hole subbands.

The phonon-assisted interband tunneling current $J_{\mathrm{ph}}$ is modeled according to the approach described in [26]. This approach, in fact, can be applied for different gas dimensionalities and arbitrary potential profiles in the structure. Now, $J_{\mathrm{ph}}$ is calculated using 


$$
\begin{aligned}
J_{\mathrm{ph}} & =\frac{-2 q}{h} \int \mathrm{d} E\left\{\left[f_{v}(E)\left(1-f_{c}\left(E-E_{\mathrm{ph}}\right)\right)\left(n_{\mathrm{B}}\left(E_{\mathrm{ph}}\right)+1\right)-f_{c}\left(E-E_{\mathrm{ph}}\right)\left(1-f_{v}(E)\right) n_{\mathrm{B}}\left(E_{\mathrm{ph}}\right)\right] T_{v}^{e m}(E)\right. \\
& \left.+\left[f_{v}(E)\left(1-f_{c}\left(E+E_{\mathrm{ph}}\right)\right) n_{\mathrm{B}}\left(E_{\mathrm{ph}}\right)-f_{c}\left(E+E_{\mathrm{ph}}\right)\left(1-f_{v}(E)\right)\left(n_{\mathrm{B}}\left(E_{\mathrm{ph}}\right)+1\right)\right] T_{v}^{\mathrm{abs}}(E)\right\}
\end{aligned}
$$

where $E_{\mathrm{ph}}$ is the phonon energy for a given material and $n_{\mathrm{B}}(E)$ is the Bose-Einstein function. Transmission probability is calculated as follows:

$$
\begin{aligned}
& T_{v}^{\text {abs, em }}(E)=\Omega\left|M_{k_{0}}^{\prime}\right|^{2} \\
& \quad \times \sum_{\substack{v=h h / l h \\
c=\Gamma}} \int \mathrm{d} z A_{v, k}(z, E) A_{c, n}\left(z, E \pm E_{\mathrm{ph}}\right),
\end{aligned}
$$

where the term $\Omega\left|M_{k_{0}}^{\prime}\right|^{2}$ describes the phonon coupling and $A_{v, k}, A_{c, n}$ are the spectral functions of $2 \mathrm{D}$ gases. The thermal generation-recombination processes are also included in the calculations based on the SRH model [27].

\section{Simulation results}

Numerical simulations were performed for $\mathrm{Si}$, InAs and Ge channel materials. Nominal simulation parameters used in this work are: channel doping level $N_{A}=10^{15} \mathrm{~cm}^{-3}$, body thickness $t=10$, 15, $20 \mathrm{~nm}$, gate overlap length $L=50 \mathrm{~nm}, \mathrm{HfO}_{2}$ as the gate oxide of thickness $t_{\mathrm{ox}}=3 \mathrm{~nm}$ and the relative dielectric permittivity $\varepsilon_{r}=22$, bottom gate work function $\Phi_{\mathrm{BG}}=5.6 \mathrm{eV}$ (unless otherwise indicated), bottom gate voltage $V_{\mathrm{BGS}}=0 \mathrm{~V}$. The appropriate bottom gate work function ensures that $2 \mathrm{DHG}$ is induced at zero bias and only the top gate voltage $V_{\text {TGS }}$ is varied in simulations. The top gate work function $\Phi_{\mathrm{TG}}$ is adjusted for different structures in a way that ensures the threshold voltage being set at relatively low values.

Depending on the material type, e.g., the semiconductor of the direct or indirect bandgap, the different tunneling current models are used to calculate the current-voltage characteristics. Schrödinger's equation is solved for heavy and light holes in $\mathrm{Si}$, InAs and Ge, and $\Gamma$-valley electrons in InAs, $\Delta$-valley electrons in $\mathrm{Si}, \Gamma$ - and $L$-valley electrons in Ge. In Figs. 2-4 we show the simulated $I-V$ characteristics for these materials for various values of the channel thickness. The phonon energy and phonon coupling term have been taken from [28]. For the $\langle 100\rangle$ crystal orientation, the effective masses in valleys of $\mathrm{Si}$ and Ge were calculated according to [29]. Simulation parameters for different materials used in this work are presented in Table I.

In Fig. 2, we show the simulated $I-V$ characteristics of Si EHB TFET for various channel thicknesses at $V_{\mathrm{DS}}=0.5 \mathrm{~V}$. In the case of the Si channel, the phonon-assisted interband tunneling current between the $\Gamma$ point in the valence band and the $\Delta$ point in the conduction band constitutes a drain-source current. In general, an increase in

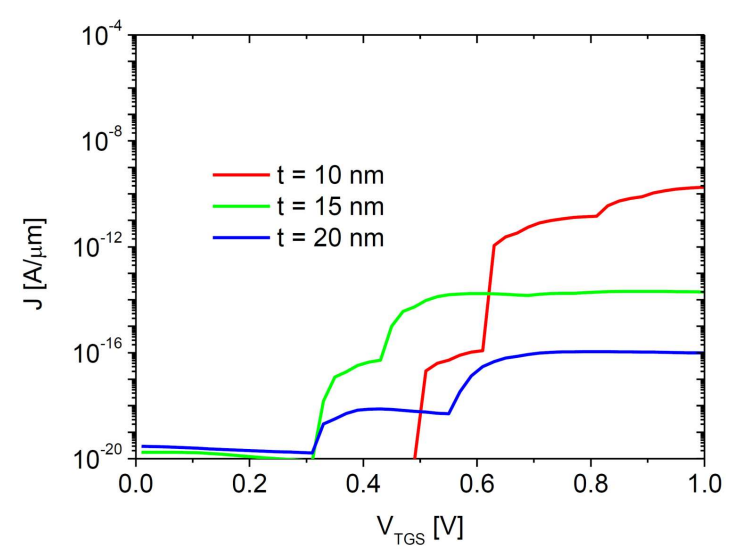

Fig. 2. Si EHB TFET current-voltage characteristics for various channel thickness $V_{\mathrm{DS}}=0.5 \mathrm{~V}$, top gate work function $\Phi_{\mathrm{TG}}=4.0 \mathrm{eV}$.

TABLE I

Simulation parameters of the investigated material with energy band gap (indirect for $\Delta$ point in $\mathrm{Si}$ and $L$ point in Ge, direct for $\Gamma$ point in Ge and InAs), heavy and light hole effective masses $\left(m_{h h}\right.$ and $m_{h l}$ ), $\Gamma$-valley electron effective mass (for InAs and $\mathrm{Ge})$, and transverse and longitudinal effective masses $(\Delta$-valley in $\mathrm{Si}$ and $L$-valley in $\mathrm{Ge})$.

\begin{tabular}{c|c|c|c}
\hline \hline & $\mathrm{Si}$ & $\mathrm{InAs}$ & $\mathrm{Ge}$ \\
\hline$E_{G}[\mathrm{eV}]$ & $1.12 @ \Delta$ & $0.354 @ \Gamma$ & $0.802 @ \Gamma$ \\
& & & $0.658 @ L$ \\
$m_{h h}$ & $0.49 m_{0}$ & $0.41 m_{0}$ & $0.33 m_{0}$ \\
$m_{h l}$ & $0.16 m_{0}$ & $0.026 m_{0}$ & $0.043 m_{0}$ \\
$m_{\Gamma}$ & - & $0.023 m_{0}$ & $0.042 m_{0}$ \\
$m_{t}$ & $0.19 m_{0} @ \Delta$ & - & $0.0815 m_{0} @ L$ \\
$m_{l}$ & $0.916 m_{0} @ \Delta$ & - & $1.59 m_{0} @ L$
\end{tabular}

the channel thickness results in a reduction of the tunneling current level due to the lower transmission probability. It also changes the gate voltage at which the hole and electron energy levels are aligned. The alignment of the energy levels results in a sudden current increase due to the phononassisted tunneling between the 2DHG and 2DEG energy levels. At $V_{\mathrm{TGS}}>0.6 \mathrm{~V}$, the highest current is observed for the smallest channel thickness. For thicker structures, we observe a higher threshold voltage at which the tunneling current starts flowing. For the Si channel structure, the current levels are very low due to the relatively large indirect bandgap. At low top gate voltage values, the current is limited by the SRH generation component. 


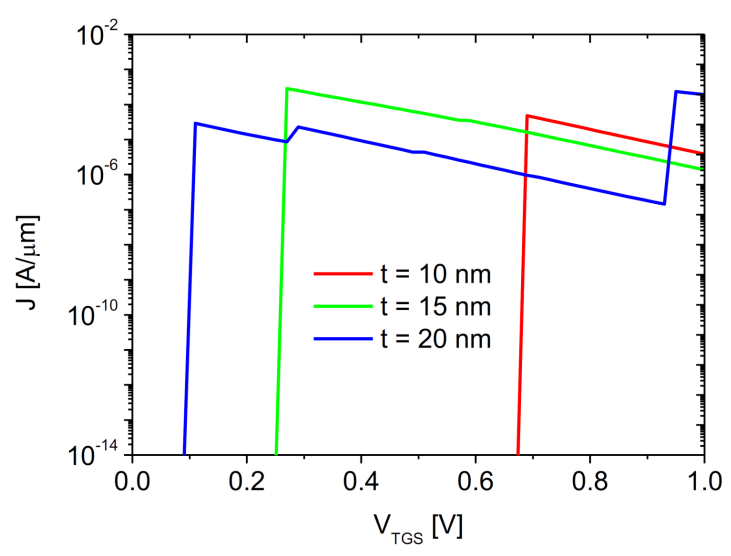

Fig. 3. InAs EHB TFET current-voltage characteristics for various channel thickness $V_{\mathrm{DS}}=0.5 \mathrm{~V}$, top gate work function $\Phi_{\mathrm{TG}}=4.3 \mathrm{eV}$, bottom gate work function $\Phi_{\mathrm{BG}}=5.0 \mathrm{eV}$.

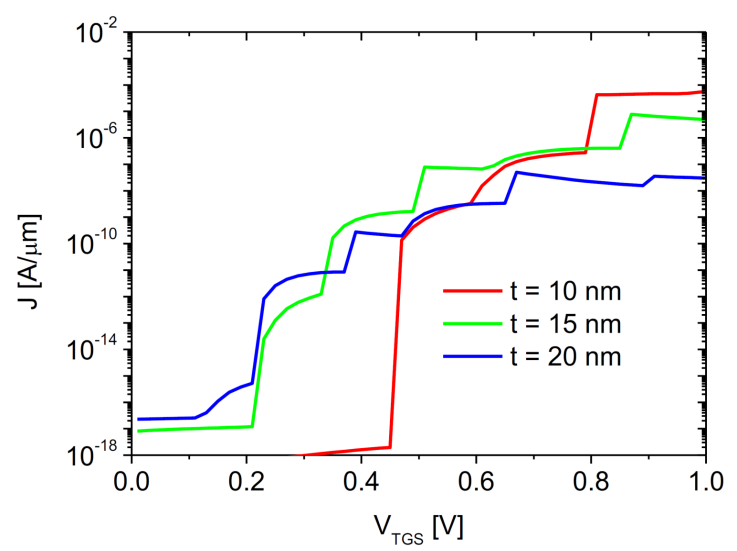

Fig. 4. Ge EHB TFET current-voltage characteristics for various channel thickness, $V_{\mathrm{DS}}=0.5 \mathrm{~V}$, top gate work function $\Phi_{\mathrm{TG}}=4.0 \mathrm{eV}$.

For InAs EHB TFET, direct interband tunneling occurs and a very sharp current rise (see Fig. 3) can be observed due to the small direct bandgap of the channel material. When the first electron and hole subband energy levels in quantum wells are aligned, the direct tunneling current starts flowing between 2DHG and 2DEG, causing a very sharp turn-on. A further increase of the gate voltage $V_{\mathrm{TGS}}$ results in a decrease of the tunneling current as a consequence of the decreasing overlap of the wave functions for the corresponding energy levels [10]. Then, the quantum wells become narrower, and wave functions are pushed towards the semiconductor/dielectric interface. The channel thickness strongly influences the electrostatics of the structure and hence, it also changes the $V_{\mathrm{TGS}}$ values at which tunneling between the different subbands occurs.

In Fig. 4, we show the simulated current-voltage characteristics for Ge EHB TFETs of various channel thicknesses, at $V_{\mathrm{DS}}=0.5 \mathrm{~V}$. The increase of the channel thickness results in a reduction of the tunneling current due to the lower transmission

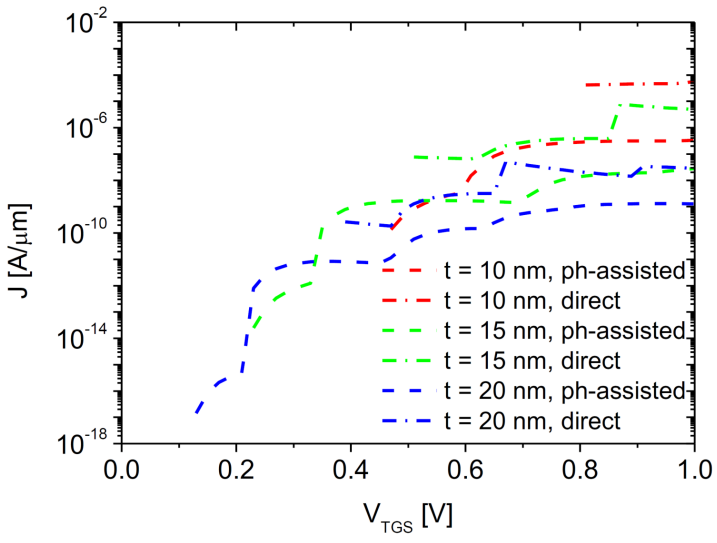

Fig. 5. Comparison of direct and phonon-assisted tunneling currents for Ge EHB TFET and various channel thicknesses, $V_{\mathrm{DS}}=0.5 \mathrm{~V}$, top gate work function $\Phi_{\mathrm{TG}}=4.0 \mathrm{eV}$.

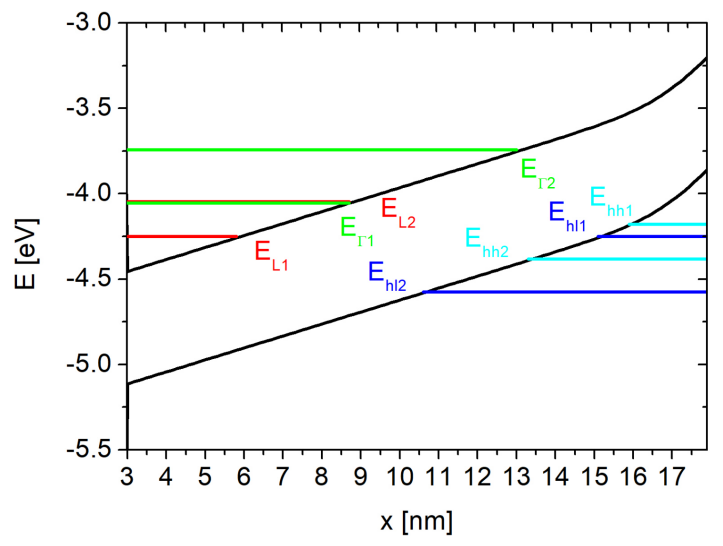

Fig. 6. Ge EHB TFET band diagram and energy levels in the cross-section of the channel for $t=15 \mathrm{~nm}, V_{\mathrm{DS}}=0.5 \mathrm{~V}, V_{\mathrm{GS}}=0.3 \mathrm{~V}$.

probability. It also changes the gate voltage values at which the hole and electron subband energy levels are aligned. Very sharp current jumps are observed at higher $V_{\mathrm{TGS}}$ voltages due to the direct interband tunneling. In Ge, the energy difference between the direct and indirect bandgaps is relatively small. In relation to this fact, the Ge EHB TFET current is a sum of phonon-assisted and direct tunneling currents and is dominated by the latter for a sufficiently high $\mathrm{V}_{\text {TGS }}$.

A comparison of direct and phonon-assisted tunneling current components for the Ge EHB TFETs of different channel thickness is shown in Fig. 5. One can observe that the phonon-assisted current starts flowing at lower values of $V_{\mathrm{TGS}}$ for thicker channels. For a sufficiently high value of the top gate voltage $\mathrm{V}_{\text {TGS }}$ one can observe that direct interband tunneling is also possible due to the alignment of hole subband energy levels and $\Gamma$-valley electron energy levels. The channel thickness has a pronounced effect on the electrostatics in the structure, and hence on the current-voltage characteristics. Note that the thinner channel results in 


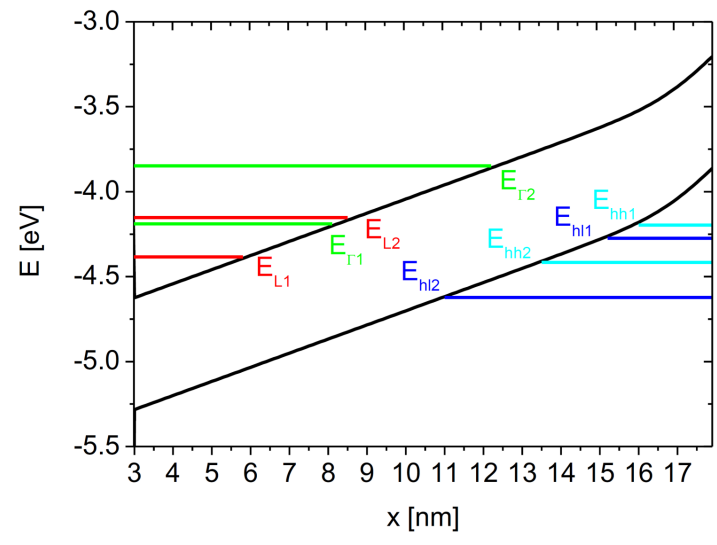

Fig. 7. Ge EHB TFET band diagram and energy levels in the cross-section of the channel for $t=15 \mathrm{~nm}, V_{\mathrm{DS}}=0.5 \mathrm{~V}, V_{\mathrm{GS}}=0.5 \mathrm{~V}$.

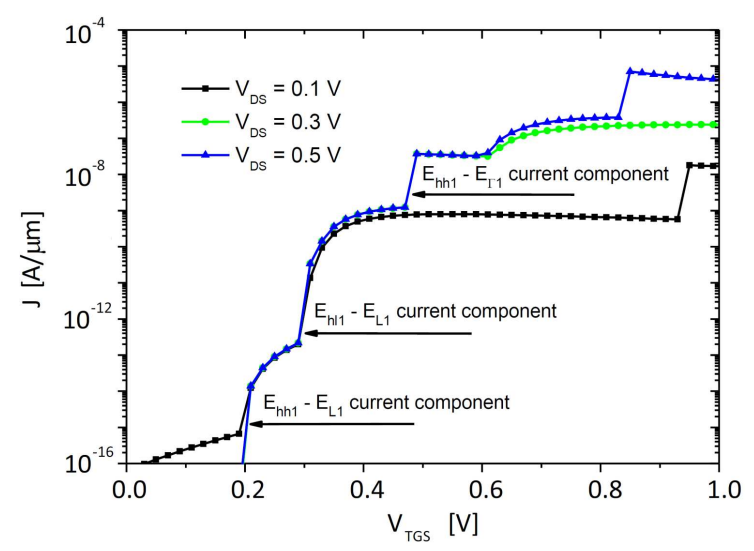

Fig. 8. Ge EHB TFET current-voltage characteristics for $t=15 \mathrm{~nm}$ and different $V_{\mathrm{DS}}$ values.

higher current values, both for phonon-assisted and direct tunneling, but also in higher threshold voltage values at which the interband tunneling current starts flowing. It is related to the width of the quantum well. For thin structures, quantum wells are narrower and the subband energy levels are higher. The quantum wells are wider for thicker structures, and the energy levels are lower in the respective bands, resulting in a lower threshold voltage.

In Figs. 6 and 7, the Ge EHB TFET band diagram and energy levels in the cross-section (band energy as a function of position $x$ ) of the channel are shown for $t=15 \mathrm{~nm}, V_{\mathrm{DS}}=0.5 \mathrm{~V}$ and different $V_{\mathrm{TGS}}$ values. Energy levels of heavy holes $E_{h h}$, light holes $E_{h l}, \Gamma$-valley electrons $E_{\Gamma}$ and $L$-valley electrons $E_{L}$ are indicated. For $V_{\mathrm{TGS}}=0.3 \mathrm{~V}$ one can see that two sub-band energy pairs $E_{h h 1}-E_{L 1}$ and $E_{h l 1}-E_{L 1}$ can contribute to the total tunneling current (heavy/light holes and $L$-valley electrons).

Thus, only the phonon-assisted tunneling current can be observed. An increase of the top gate voltage to $0.5 \mathrm{~V}$ results in an alignment of the sub-band energy pair at $\Gamma$ point $\left(E_{h h 1}-E_{\Gamma 1}\right)$, enabling the direct interband tunneling.

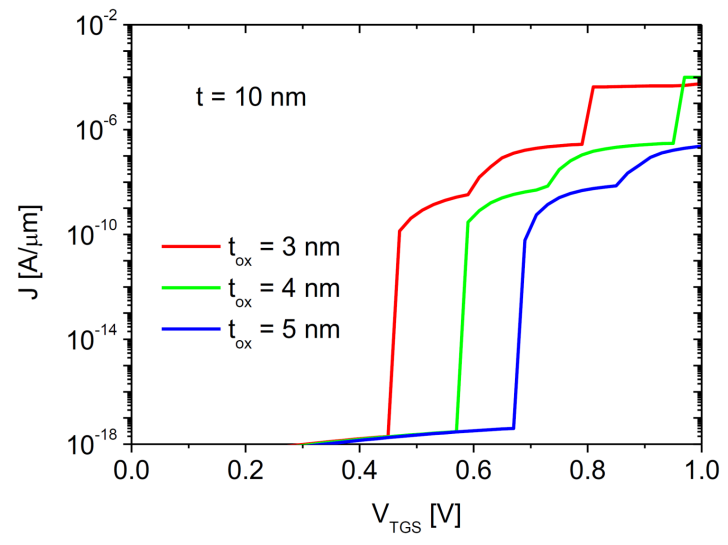

Fig. 9. Ge EHB TFET current-voltage characteristics for various top gate oxide thickness, $V_{\mathrm{DS}}=0.5 \mathrm{~V}$, top gate work function $\Phi_{\mathrm{TG}}=4.0 \mathrm{eV}$.

In Fig. 8, the current-voltage characteristics as a function of $V_{\mathrm{TGS}}$ for different $V_{\mathrm{DS}}$ and body thickness $t=15 \mathrm{~nm}$ are shown. The arrows mark places where the tunneling between the labeled subband energy pairs starts flowing at $V_{\mathrm{DS}}=0.5 \mathrm{~V}$. The result for this case is consistent with the band diagrams shown in Figs. 6 and 7. One can also see that the current level at $V_{\text {TGS }}>0.5 \mathrm{~V}$ strongly varies for different $V_{\mathrm{DS}}$ values. A change in $V_{\mathrm{DS}}$ results in a change of electrostatics in the structure, position of quantized energy levels, and therefore the tunneling current level.

In Fig. 9, the transfer current-voltage characteristics for Ge EHB TFET of channel thickness $t=10 \mathrm{~nm}$ and various top-gate oxide thickness values $t_{\mathrm{ox}}$ are shown. A thicker oxide occurs in a higher threshold voltage. A higher gate voltage value is needed to align the sub-band energy levels to start the flow of the interband tunneling current. A threshold voltage of the device can be adjusted by using the gate material with a proper work function for a given transistor geometry.

\section{Conclusions}

In this work, a study of the current-voltage characteristics of an electron-hole bilayer tunnel field effect transistor by means of a quantum mechanical modeling is presented. The model takes into account direct and phonon-assisted interband tunneling. The impact of the semiconductor band structure is especially considered in the example of $\mathrm{Si}, \mathrm{Ge}$, and InAs channels. In general, the paper shows that the EHB TFET structure can exhibit very sharp turn-on transfer characteristics due to the interband tunneling as the transport mechanism and due to the step-like distributions of densities of states in the 2D electron/hole gas formed in quantum wells induced in the vicinity of the top and bottom gates. Therefore, it is a promising structure for low-power circuits. 


\section{Acknowledgments}

This work was supported by the National Science Centre, Poland, through project No. 2018/31/N/ST7/01147 entitled "Study of the interband tunneling phenomenon between low dimensional carrier gases in tunnel field-effect transistor".

\section{References}

[1] A.M. Ionescu, H. Riel, Nature 479, 329 (2011).

[2] U.E. Avci, D.H. Morris, I.A. Young, IEEE J. Electron Dev. Soc. 3, 88 (2015).

[3] L. Lattanzio, L. De Michielis, A.M. Ionescu, Solid State Electron 74, 85 (2012).

[4] H. Lu, A. Seabaugh, IEEE J. Electron Dev. Soc. 2, 44 (2014).

[5] L. Lattanzio, L. De Michielis, A.M. Ionescu, in: Proc. European Solid-State Device Research Conference (ESSDERC), 2011, p. 259.

[6] H. Lüth, Acta Phys. Pol. A 90, 667 (1996).

[7] S. Agarwal, E. Yablonovitch, in: 69th Device Research Conf., 2011, p. 199.

[8] S. Agarwal, E. Yablonovitch, arXiv:1109.0096v3 (2014).

[9] C. Alper, J.L. Padilla, P. Palestri, A.M. Ionescu, in: 46th European SolidState Device Research Conf. (ESSDERC), 2016, p. 307.

[10] L. Lattanzio, N. Dagtekin, L. De Michielis, A.M. Ionescu, IEEE Trans. Electron Dev. 59, 2932 (2012).

[11] C. Alper, L. Lattanzio, L. De Michielis, P. Palestri, L. Selmi, A.M. Ionescu, IEEE Trans. Electron Dev. 60, 2754 (2013).

[12] C. Alper, P. Palestri, J.L. Padilla, A. Gnudi, R. Grassi, E. Gnani, M. Luisier, A.M. Ionescu, in: EUROSOI-ULIS 20152015 Jt. Int. EUROSOI Work. Int. Conf. Ultim. Integr. Silicon, 2015, p. 141.

[13] J.L. Padilla, C. Alper, A. Godoy, F. Gamiz, A.M. Ionescu, IEEE Trans. Electron Dev. 62, 3560 (2015).
[14] P. Wisniewski, B. Majkusiak, IEEE Trans. Electron Dev. 67, 2738 (2020).

[15] J.L. Padilla, C. Medina-Bailón, M. Rupakula, C. Alper, C. Sampedro, F. Gámiz, A.M. Ionescu, in: Jt. Int. EUROSOI Work. Int. Conf. Ultim. Integr. Silicon, (EUROSOI-ULI), 2018.

[16] J.L. Padilla, C. Medina-Bailon, C. Alper, F. Gamiz, A.M. Ionescu, Appl. Phys. Lett. 112, 182101 (2018).

[17] Z. Ahangari, J. Comput. Electron 19, 1485 (2020).

[18] A. Revelant, A. Villalon, Y. Wu, A. Zaslavsky, C. Le Royer, H. Iwai, S. Cristoloveanu, IEEE Trans. Electron Dev. 61, 2674 (2014).

[19] K. Kato, H. Matsui, H. Tabata, M. Takenaka, S. Takagi, Appl. Phys. 125, 195701 (2019).

[20] K. Kato, H. Matsui, H. Tabata, M. Takenaka, S. Takagi, IEEE J. Electron Dev. Soc. 7, 1201 (2019).

[21] K. Kato, K.W. Jo, H. Matsui, H. Tabata, T. Mori, Y. Morita, T. Matsukawa, M. Takenaka, S. Takagi, IEEE Trans. Electron Dev. 67, 1880 (2020).

[22] J. Bardeen, Phys. Rev. Lett. 6, 57 (1961).

[23] J.M. Bigelow, J.P. Leburton, IEEE Trans. Electron Dev. 41, 125 (1994).

[24] M. Yamanishi, I. Suemune, Jpn. J. Appl. Phys. 23, L35 (1984).

[25] M. Asada, A. Kameyama, Y. Suematsu, IEEE J. Quant. Electron. 20, 745 (1984).

[26] W. Vandenberghe, B. Sorée, W. Magnus, M.V. Fischetti, J. Appl. Phys. 109, 124503 (2011).

[27] W. Shockley, W.T. Read, Phys. Rev. 87, 835 (1952).

[28] K.-H. Kao, A.S. Verhulst, W.G. Vandenberghe, B. Soree, G. Groeseneken, K. De Meyer, IEEE Trans. Electron Dev. 59, 292 (2012).

[29] F. Stern, W.E. Howard, Phys. Rev. 163, 816 (1967). 